\title{
The Obesity Paradox in Recurrent Attacks of Gout in Observational Studies: Clarification and Remedy
}

DOI:

10.1002/acr.22954

\section{Document Version}

Accepted author manuscript

Link to publication record in Manchester Research Explorer

\section{Citation for published version (APA):}

Nguyen, U. S. D. T., Zhang, Y., Louie-Gao, Q., Niu, J., Felson, D., Lavalley, M., \& Choi, H. K. (2017). The Obesity Paradox in Recurrent Attacks of Gout in Observational Studies: Clarification and Remedy. Arthritis Care and Research, 69(4), 561-566. https://doi.org/10.1002/acr.22954

\section{Published in:}

Arthritis Care and Research

\section{Citing this paper}

Please note that where the full-text provided on Manchester Research Explorer is the Author Accepted Manuscript or Proof version this may differ from the final Published version. If citing, it is advised that you check and use the publisher's definitive version.

\section{General rights}

Copyright and moral rights for the publications made accessible in the Research Explorer are retained by the authors and/or other copyright owners and it is a condition of accessing publications that users recognise and abide by the legal requirements associated with these rights.

\section{Takedown policy}

If you believe that this document breaches copyright please refer to the University of Manchester's Takedown Procedures [http://man.ac.uk/04Y6Bo] or contact uml.scholarlycommunications@manchester.ac.uk providing relevant details, so we can investigate your claim.

\section{OPEN ACCESS}


The Obesity Paradox in Recurrent Attacks of Gout in Observational Studies: Clarification and Remedy

\title{
Uyen-Sa D. T. Nguyen ${ }^{1,2}$, Yuqing Zhang ${ }^{2}$, Qiong Louie-Gao ${ }^{2}$, Jingbo Niu ${ }^{2}$
}

\author{
David T. Felson ${ }^{2}$, Michael P. LaValley ${ }^{3}$, Hyon K. Choi ${ }^{2,4}$
}

${ }^{1}$ University of Massachusetts Medical School, Department of Orthopedics and Physical Rehabilitation; ${ }^{2}$ Boston University School of Medicine, Department of Medicine, Clinical Epidemiology Research \& Training Unit; ${ }^{3}$ Boston University School of Public Health, Department of Biostatistics; ${ }^{4}$ Massachusetts General Hospital, Harvard Medical School Department of Rheumatology

\section{Corresponding Author: Uyen-Sa D. T. Nguyen, DSc, MPH

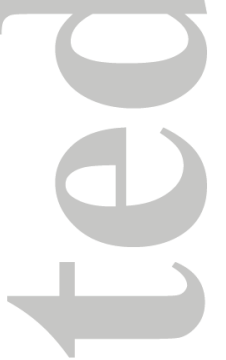 \\ University of Massachusetts Medical School \\ Department of Orthopedics and Physical Rehabilitation \\ 55 Lake Ave. North, S4-312, Worcester, MA 01655, USA \\ Phone: 774-455-4487 (as of 04/26/16); Fax: 508-856-2581 \\ Email: uyensa.nguyen@umassmed.edu}

Grants: This study is supported in part by grants from the Arthritis Foundation, Rheumatology

Research Foundation Innovative Research Award, NIH NIAMS K01AR064351, R21AR068493, and P60AR047785.

Word Count: 3133

Tables: 3

Running Title: The Obesity Paradox in Recurrent Attacks of Gout

Appendices: Supplemental 2 Tables/2 Figures

This article has been accepted for publication and undergone full peer review but has not been through the copyediting, typesetting, pagination and proofreading process which may lead to differences between this version and the Version of Record. Please cite this article as an 'Accepted Article', doi: 10.1002/acr.22954

(C) 2016 American College of Rheumatology

Received: Feb 10, 2016; Revised: May 10, 2016; Accepted: Jun 07, 2016 


\begin{abstract}
Objective: Obesity is strongly associated with incident gout risk; its association with risk of recurrent gout attacks has been null or weak, constituting an obesity paradox. We sought to demonstrate and overcome the methodologic issues associated with the obesity paradox for risk of recurrent gout attacks.
\end{abstract}

Methods: Using the MRFIT database, we decomposed the total effect of obesity into its direct and indirect (i.e., mediated) effects using marginal structural models. We also estimated the total effect of BMI change from baseline among incident gout patients.

Results: Of 11,816 gout-free subjects at baseline, we documented 408 incident gout cases, with 132 developing recurrent gout attacks over a 7-year follow-up. The adjusted odds ratio (OR) for incident gout among obese individuals was 2.6, while that for recurrent gout attacks among gout patients was 0.98 (i.e., the obesity paradox). These ORs correlated well with the ORs for the indirect and direct effects of obesity on risk of recurrent gout attacks (i.e., 2.83 and 0.98, respectively). Compared with no BMI change, the OR of losing vs. gaining $>5 \%$ of baseline BMI was 0.61 and 1.60 for recurrent gout attacks, respectively ( $\mathrm{P}$ for trend $<0.01$ ), suggesting a dose-response association.

Conclusion: The obesity paradox for risk of recurrent gout attacks is explained by the absence of the direct effect, which is often measured in conventional analyses and misinterpreted as the intended total effect of interest. In contrast, the BMI change analysis correctly estimated the intended total effect of BMI, and revealed a dose-response relationship.

Keywords: Gout, Obesity, Mediation Analysis, Obesity Paradox, Total Effect, Direct and Indirect Effects 


\section{Significance and Innovations}

- The obesity paradox for risk of recurrent attacks of gout (i.e., the null or weak impact of BMI on risk of recurrent attacks of gout) is likely due to a mismatched study design and analytic approach.

- In contrast, the analysis of BMI change can overcome this methodologic limitation.

- BMI loss decreased the risk of recurrent attacks of gout, whereas BMI gain increased the risk of recurrent attacks of gout.

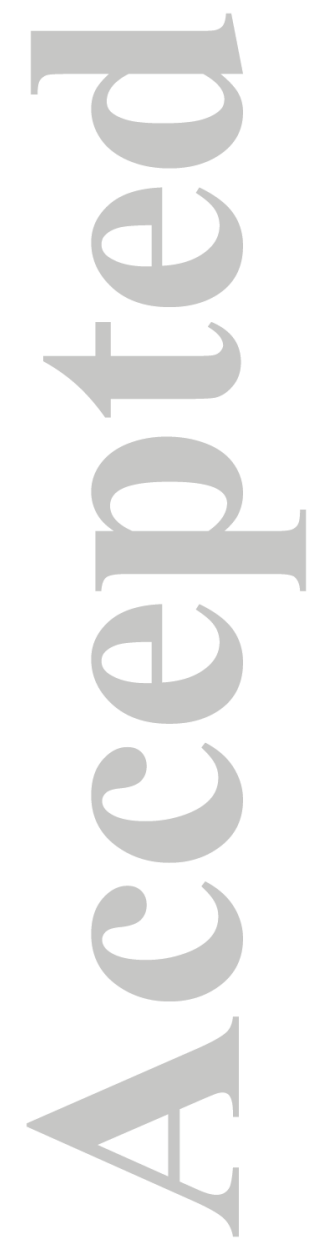

John Wiley \& Sons, Inc. 


\section{INTRODUCTION}

Gout is the most common form of inflammatory arthritis in the US, affecting 8.3 million adults ${ }^{1}$. Gout is causally linked to hyperuricemia, and acute flares constitute one of the most painful conditions experienced by humans. Among many known risk factors for gout, obesity is the strongest modifiable risk factor for incident gout ${ }^{2,3}$. However, unlike its impact on incident gout, study findings for the risk of recurrent attacks of gout have been paradoxical. For example, a prospective study found a null association between BMI and the risk of recurrent attacks of gout, ${ }^{4}$ and a retrospective electronic medical record-based study found a substantially smaller magnitude of association than that with the risk of incident gout ${ }^{5}$. Such paradoxical associations in the analysis of risk factors are not unique to this context, and have been observed in studies of sequela (or recurrent events) in many other disease contexts as well, including the smoking and obesity paradox for mortality risk in coronary artery disease or chronic obstructive pulmonary disease; the relation of smoking, obesity and the Apo E4 risk allele to progression of rheumatoid arthritis, osteoarthritis and cognitive function decline; as well as the patent foramen ovale paradox for recurrent stroke ${ }^{6}$.

Although biological explanations for these counterintuitive results may exist, methodological flaws provide an enticing alternative explanation for the seemingly paradoxical phenomena observed in these contexts. One methodologic explanation is the mismatch between the study question of interest and the study design/analytic approach, particularly between the intended effect measure (i.e., total effect) and the effect measure (i.e., direct effect) actually obtained ${ }^{6,7}$. Thus, the objectives of our study were to illustrate the obesity and recurrent attacks of gout paradox by clarifying the possible underlying mechanism and demonstrating an appropriate 
remedy. To that effect, we employed mediation analysis to clarify this paradoxical phenomenon, and we analyzed the impact of change in BMI after gout diagnosis on the risk of recurrent attacks of gout as a remedy to appropriately assess the total effect of obesity.

\section{MATERIALS AND METHODS}

\section{Study Population}

We used data from the Multiple Risk Factor Intervention Trial (MRFIT) to assess BMI and BMI change on the risk of incident and recurrent attacks of gout. Detailed descriptions of MRFIT have been published elsewhere ${ }^{8-11}$. In brief, 361,662 men were screened between 1973 and 1976 for eligibility at 22 clinical centers. Eligibility criteria included being in the upper $15 \%$ of the Framingham Heart Study Risk Score ${ }^{12}$, which was based on the participant's smoking, hyperlipidemia, and hypertension status. Of those deemed to be eligible, 12,866 men between 35 and 57 years of age were randomly assigned to either a special intervention group $(n=6428)$ or the usual care group $(n=6428)$. The MRFIT intervention program included smoking cessation, weight reduction, increased physical activity, and anti-hypertensive treatment. The men were followed annually over 7 years (total of 84 months), with a follow-up rate of over $90 \%{ }^{13}$. The current study population consisted of those without a diagnosis of gout at baseline.

\section{Assessment of body mass index (BMI)}

Height and weight were measured at baseline and every year thereafter at regularly scheduled clinic visits ${ }^{11}$. Body mass index (BMI) was calculated as weight in kilograms divided by the square of height in meters $\left(\mathrm{kg} / \mathrm{m}^{2}\right)$, and was further categorized as either $\geq 30 \mathrm{~kg} / \mathrm{m}^{2}$ (obese), 25 $29.9 \mathrm{~kg} / \mathrm{m}^{2}$ (overweight), or $<25 \mathrm{~kg} / \mathrm{m}^{2}$ (normal). 


\section{Assessment of gout}

We used a case definition of gout based on participants' positive answer to the question, "Have you been told by your physician that you have gout?" at the baseline visit. At subsequent visits, gout was defined as occurring in participants who answered affirmatively to the follow-up question, "Based on the medical history over the past 12 months, did you have gout attack?" These definitions have been successfully used in a number of gout studies ${ }^{14-16}$.

\section{$\underline{\text { Assessment covariates }}$}

At baseline and every year thereafter subjects provided demographic information, including age, number of years of education (with those having completed college defined as those with a level of education $\geq 16$ years vs. not), and a 24 -hour dietary recall which included information on alcohol intake (defined as drinks per day: $0, \leq 1,>1$ ) and coffee intake (defined as cups per day: $0, \leq 1,>1)$. Subjects also provided a detailed medical history and underwent a full physical examination. Procedures and protocols for each visit have been described in detail previously $^{17,18}$. Blood pressure measurements were recorded as the average of two measurements. Hypertension was defined as systolic blood pressure $\geq 140 \mathrm{mmHg}$, diastolic blood pressure $\geq 90 \mathrm{mmHg}$, or use of antihypertensive medications at each visit.

\section{$\underline{\text { Statistical Analysis }}$}

To clarify the paradoxical phenomenon of obesity on the risk of recurrent attacks of gout, we partitioned the total effect (see Appendix text and Figures) of BMI on the risk of recurrent attacks of gout into direct (non-mediated) and indirect (mediated through the development of incident gout) effects using marginal structural models (MSM), with a pooled logistic regression 
model as a discrete-time method ${ }^{19,20}$. The exposure variable was baseline BMI, the intermediate variable was the occurrence of the first gout attack (i.e., incident gout), and recurrent attacks of gout was the outcome of interest. Inclusion of follow-up time continued until either the occurrence of the second gout attack, loss to follow-up, or the end of the study period, whichever came first. We obtained estimates of the indirect effect of obesity on the risk of recurrent attacks of gout (i.e., the obesity effect which was through its effect on incident gout), and direct effect of obesity on the risk of recurrent attacks of gout (i.e., the obesity effect that did not involve its effect on incident gout $)^{20}$. Our multivariable model adjusted for age, education, alcohol and coffee intake, presence of hypertension, and diuretic use at baseline.

We then demonstrated a remedy to estimate the total effect of BMI on the risk of recurrent attacks of gout among gout patients. First, we used BMI change before and after the incident gout attack as an exposure variable and assessed its relation to the risk of recurrent attacks of gout. Study subjects included in this analysis consisted of those with incident gout. These subjects were followed until either they had a recurrent attack of gout attack, were lost to followup, or the study ended. Nested in this cohort, we conducted a risk set sampling case-control study where cases had recurrent attacks of gout at the end of the biennial follow-up period. For each case of recurrent attacks of gout, we randomly selected up to 10 controls who did not experience a recurrent attack of gout at the time of case diagnosis (i.e., index time), matching by the time of the first gout attack (matched risk sets). We calculated the percent of BMI change using the BMI just prior to the occurrence of the first gout attack. We categorized the percent of BMI change according to five levels: loss $>5 \%$, loss of $3.6 \%$ to $5.0 \%, \pm 3.5 \%$ (stable), gain of $3.6 \%$ to $5.0 \%$, and gain $>5 \%$. Note that a $5 \%$ change is approximately equal to $0.15 \mathrm{~kg}$ weight 
loss or gain for a person of height $1.74 \mathrm{M}$, and $3.5 \%$ is approximately equal to $0.1 \mathrm{~kg}$. We used conditional logistic regression to estimate the effect of BMI change on the risk of recurrent attacks of gout, adjusting for age, education, alcohol and coffee intake, presence of hypertension and diuretic use 12 months prior to the incident gout attack. We tested the dose-response relationship between BMI change and the risk of recurrent attacks of gout by entering the median value of each BMI change category in the multivariable conditional logistic regression model.

\section{RESULTS}

Of 11,896 MRFIT participants without gout at baseline, $21 \%$ of them had a normal BMI, 55\% were overweight, and 23\% were obese. As shown in Table 1, compared with the normal BMI group, those in the obese group tended to be less educated and have a lower proportion of consumption of more than one alcoholic drink per day, but tended to have a higher proportion of hypertension and diuretic use.

During the 7-year follow up period, 408 participants developed incident gout, among which 132 reported recurrent attacks of gout. Baseline obesity was associated with a 2.6 increased risk of incident gout in the entire population (adjusted $\mathrm{OR}=2.60,95 \% \mathrm{CI}=1.90,3.57$ ), but not with recurrent attacks of gout among patients with incident gout (adjusted $\mathrm{OR}=0.98,95 \% \mathrm{CI}=0.53$, 1.81), confirming the presence of an obesity paradox in this dataset (see Web appendix supplemental Tables 1 and 2).

Results to clarify this paradoxical phenomenon using mediation analyses with inverse probability weighting of MSM are shown in Table 2. Compared with people of normal BMI, the total effect 
of obesity on the risk of recurrent attacks of gout (in the entire study population regardless of incident gout status) was almost 3 -fold (adjusted $\mathrm{OR}=2.75,95 \% \mathrm{CI}=1.50,5.04)$. The indirect effect of obesity on the risk of recurrent attacks of gout that was mediated by incident gout was almost the same as the total effect (adjusted $\mathrm{OR}=2.83,95 \% \mathrm{CI}=2.17,3.67$ ), whereas the direct effect of obesity that was not mediated by incident gout was null (adjusted $\mathrm{OR}=0.98,95 \% \mathrm{CI}$ : $0.71,1.34)$, indicating that obesity could not have an impact on the risk of recurrent attacks of gout without going through its effect on incident gout. A similar pattern was also observed for those who were overweight.

As a remedy to resolve this paradoxical phenomenon, BMI change in relation to the risk of recurrent attacks of gout among gout patients are presented in Table 3. Compared with those whose BMI was stable, subjects who lost $>5 \%$ of their baseline BMI had a $40 \%$ decreased risk of recurrent attacks of gout, whereas those who gained $>5 \%$ of their baseline BMI had a $60 \%$ increased risk. While the individual adjusted odds ratio in each category of BMI change was not statistically significant (likely due to the relatively small sample size of the individual categories), the trend in the dose-response association between BMI change and risk of recurrent gout attacks was statistically significant (P for trend $<0.01)$.

\section{DISCUSSION}

Our objective was to provide the germane effect of obesity on risk of recurrent attacks of gout using an appropriate observational study design, and to clarify the methodologic mechanisms underlying the observed obesity paradox for the risk of recurrent attacks of gout in previous studies. Using a study design that measured the total effect of BMI change as the exposure based 
on a large clinical trial, we found that BMI change was associated with the risk of recurrent attacks of gout in a dose-responsive manner such that BMI loss decreased the risk, whereas BMI gain increased the risk of recurrent attacks of gout. In contrast, our mediation analysis found that the effect of static obesity value as the exposure on the risk of recurrent attacks of gout (as performed in previous studies ${ }^{4,5}$ ) was entirely mediated through its effect on incident gout (i.e., indirect effect). Conversely, there was no direct effect of obesity independent of its effect on incident gout, which explained why null or weak associations were found in previous studies ${ }^{4,5}$. A previous prospective cohort analysis for recurrent attacks of gout among gout patients found a null association between obesity $\left(B M I>=30 \mathrm{~kg} / \mathrm{m}^{2}\right)$ and the risk of recurrent attacks of gout $(\mathrm{OR}=1.0)^{4}$. Furthermore, a UK general population database analysis reported that obesity was weakly associated with recurrent attacks of gout $(\mathrm{RR}=1.22$ as compared to those with a normal BMI) among gout patients ${ }^{5}$. Although these studies intended to evaluate the impact of obesity on recurrent attacks of gout among gout patients, their analyses actually estimated the effect of continued obesity status since before the study baseline (and gout onset). Otherwise put, these studies estimated the direct effect of obesity (not mediated through incident gout) in the general population (as opposed to the total effect of obesity among gout patients, as intended), which was found to be null according to our results. Such evidence derived by mismatches between the study question of interest and the study design/analytic approach can be misconstrued such that although obesity is a risk factor for the development of gout, for those who have already developed gout, remaining obese does not contribute to an increased risk of recurrent attacks of gout. Thus, generating germane and intended evidence using an appropriate study design is essential for the central aim of evidence-based medicine and public health practice. 
Such evidence can be provided by a well-conducted randomized clinical trial (RCT), although it would require substantial resources. Specifically, eligible subjects in the RCT would consist of gout patients who were overweight/obese. Subjects would be randomly assigned to one group

receiving an intervention to promote weight loss and the other group would not. Then, one would be able to estimate the effect of weight loss on the risk of recurrent attacks of gout. Note that in such an RCT, any change in the exposure of interest (i.e., weight loss) would occur after the diagnosis of gout, and thus, we would be assessing the total effect of weight loss on the risk of recurrent attacks of gout. Although observational, our exposure of interest was also BMI change (from the time of the first gout attack) that occurred after the initial diagnosis of gout. Thus, in principle, this analysis was able to emulate an RCT design and was able to provide a valid estimate of the total effect of weight loss or gain on the risk of recurrent attacks of gout among gout patients. Conversely, if there were no change in BMI, it would be impossible to ascertain such a total effect, as in the case of studying baseline BMI impact on the risk of recurrent attacks of gout among gout patients, which only provided a direct effect estimate, thus creating the paradoxical phenomenon observed in prior work in gout patients.

Our BMI change results agree well with several previous studies with outcomes of serum uric acid (SUA). In a prospective 1-year follow-up of 60 individuals with severe obesity and type 2 diabetes $^{21}$, extreme weight reduction owing to bariatric surgery resulted in a $41 \%$ reduction in the proportion of patients with SUA levels higher than the usual urate-lowering therapy target $(\mathrm{SUA}=0.36 \mathrm{mmol} / \mathrm{L}[6 \mathrm{mg} / \mathrm{dL}])$. Moreover, results from a previous analysis of weight change in the MRFIT study showed that increasing weight loss compared to no weight change resulted in 
an increasingly strong likelihood of achieving the usual urate-lowering therapy $\operatorname{target}^{1}$. Similar findings were seen in a Japanese study of a dietary intervention ${ }^{22}$. Not only were SUA and other obesity-related cardiovascular risk factors lowered with weight loss as a result of a bariatric surgery intervention, but also with dietary restriction as well. In a relatively small dietary intervention study among 13 non-diabetic gout patients ${ }^{23}$, a low-calorie diet over 16 weeks achieved a weight loss of $7.7 \mathrm{~kg}$, a SUA reduction of 0.57 to $0.47 \mathrm{mmol} / \mathrm{L}$, and a reduction in monthly gout attacks from 2.1 to 0.6 . It was postulated that weight loss decreased SUA levels through increasing renal excretion of urate and decreasing urate production, thereby decreasing the risk of recurrent attacks of gout.

Several issues regarding our study design warrant discussion. First, approximately $1 / 3$ of the participants in our study with incident gout developed a recurrent gout attack over the follow up; this proportion was lower than expected. Results from an online case-crossover study among gout patients with at least one gout attack in the year prior found that $69 \%$ developed recurrent gout attacks in one year ${ }^{24}$. Since the MRFIT study was an intervention trial to reduce BMI and lower blood pressure, it is possible that these interventions also resulted in a lower incidence of recurrent gout attacks among MRFIT participants. While the proportion of recurrent gout attacks from our current study may not be generalizable to the typical population of people with existing gout, our results, which are about the biologic effect between BMI change and the risk of recurrent gout attacks, should be generalizable. Second, using the change in BMI during our study period for the assessment of recurrent attacks of gout may not have allowed a wide window of time for substantial BMI change. Despite the lack of power to indicate statistical significance for each category of percent BMI change in comparison with no change in assessing 
the risk of recurrent attacks of gout among gout patients, the test for a trend showed statistical significance. Moreover, we used self-reported gout as our outcome of interest, which may have contributed to misclassification of some cases. However, such misclassification would likely dilute the effect estimate toward the null; moreover, the same definitions have been used in prior gout studies based on MRFIT data.

In conclusion, our study demonstrated methodological issues as a possible explanation for the perceived paradox in prior studies of the impact of obesity on the risk of recurrent attacks of gout, and provided an appropriate study design to address this important issue in gout research and care. Using a proper study design and analytic method to estimate the intended germane effects, we found that BMI loss decreased the risk, whereas BMI gain increased the risk of recurrent attacks of gout.

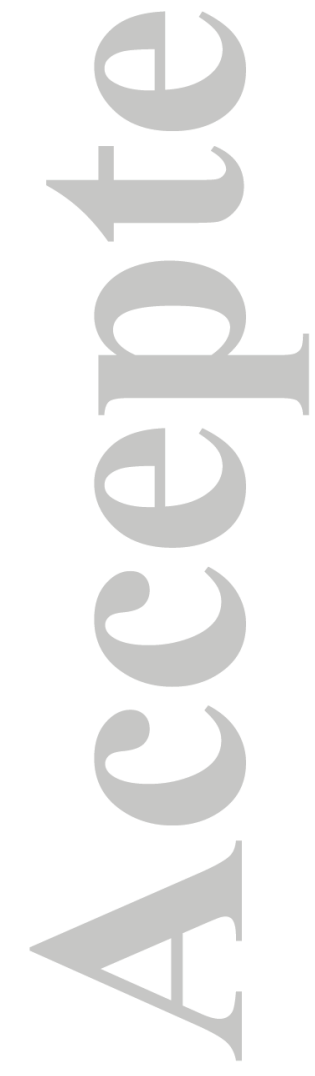




\section{Acknowledgements}

This study was performed using a limited-access data set of the Multiple Risk Factor Intervention Trial (MRFIT) Research Materials, obtained from the National Heart, Lung, and Blood Institute (NHLBI) Biologic Specimen and Data Repository Information Coordinating Center. The MRFIT was conducted and supported by the NHLBI in collaboration with the MRFIT investigators.

This current study does not necessarily reflect the opinions or views of the MRFIT or the

NHLBI, and was partly funded by the Arthritis Foundation, the American College of Rheumatology Research Foundation Innovative Research Award, NIH NIAMS K01AR064351, R21AR068493, and P60AR047785. The funders had no involvement in the study design; in the collection, analysis, or interpretation of the data; in the writing of the report; or in the decision to submit the article for publication.

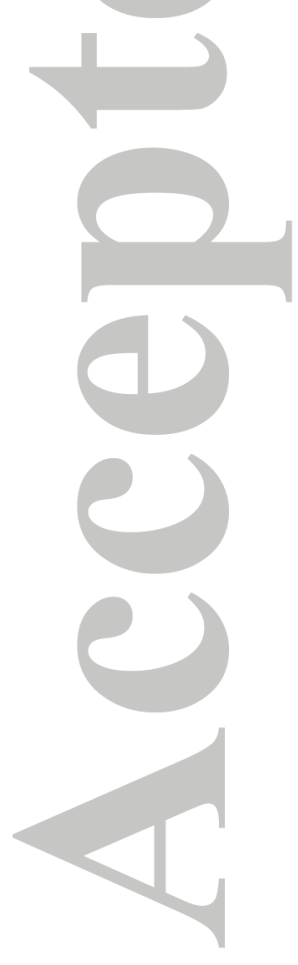




\section{REFERENCES:}

1. Zhu Y, Zhang Y, Choi HK. The serum urate-lowering impact of weight loss among men with a high cardiovascular risk profile: the Multiple Risk Factor Intervention Trial. Rheumatology (Oxford) 2010;49:2391-9.

2. Roddy E, Choi HK. Epidemiology of gout. Rheum Dis Clin North Am 2014;40:155-75.

3. Choi HK, Atkinson K, Karlson EW, Curhan G. Obesity, weight change, hypertension, diuretic use, and risk of gout in men: the health professionals follow-up study. Arch Intern Med 2005;165:742-8.

4. Zhang Y, Chaisson CE, McAlindon T, et al. The online case-crossover study is a novel approach to study triggers for recurrent disease flares. J Clin Epidemiol 2007;60:50-5.

5. Cea Soriano L, Rothenbacher D, Choi HK, Garcia Rodriguez LA. Contemporary epidemiology of gout in the UK general population. Arthritis Res Ther 2011;13:R39.

$6 . \quad$ Choi HK, Nguyen US, Niu J, Danaei G, Zhang Y. Selection bias in rheumatic disease research. Nat Rev Rheumatol 2014;10:403-12.

7. Zhang Y, Niu J, Felson DT, Choi HK, Nevitt M, Neogi T. Methodologic challenges in studying risk factors for progression of knee osteoarthritis. Arthritis Care Res (Hoboken) 2010;62:1527-32.

8. Grimm RH, Jr., Tillinghast S, Daniels K, et al. Unrecognized myocardial infarction: experience in the Multiple Risk Factor Intervention Trial (MRFIT). Circulation 1987;75:II6-8.

9. Lundberg GD. MRFIT and the goals of The Journal. JAMA 1982;248:1501.

10. Neaton JD, Grimm RH, Jr., Cutler JA. Recruitment of participants for the multiple risk factor intervention trial (MRFIT). Control Clin Trials 1987;8:41S-53S.

11. Sherwin R, Kaelber CT, Kezdi P, Kjelsberg MO, Thomas HE, Jr. The multiple risk factor intervention trial (MRFIT) II. The development of the protocol. Prev Med 1981;10:402-25.

12. Jacobs D. Hyperuricemia as a risk factor in coronary heart disease. Adv Exp Med Biol 1977;76B:231-7.

13. Krishnan E. Chronic kidney disease and the risk of incident gout among middle-aged men: a seven-year prospective observational study. Arthritis Rheum 2013;65:3271-8.

14. Gelber AC, Klag MJ, Mead LA, et al. Gout and risk for subsequent coronary heart disease. The Meharry-Hopkins Study. Archives of internal medicine 1997;157:1436-40.

15. Choi HK, De Vera MA, Krishnan E. Gout and the risk of type 2 diabetes among men with a high cardiovascular risk profile. Rheumatology (Oxford) 2008;47:1567-70.

16. Krishnan E, Baker JF, Furst DE, Schumacher HR. Gout and the risk of acute myocardial infarction. Arthritis Rheum 2006;54:2688-96.

17. Multiple risk factor intervention trial. Risk factor changes and mortality results. Multiple Risk Factor Intervention Trial Research Group. JAMA 1982;248:1465-77.

18. Gotto AM, Jr. The Multiple Risk Factor Intervention Trial (MRFIT). A return to a landmark trial. JAMA 1997;277:595-7.

19. D'Agostino RB, Lee ML, Belanger AJ, Cupples LA, Anderson K, Kannel WB. Relation of pooled logistic regression to time dependent Cox regression analysis: the Framingham Heart Study. Stat Med 1990;9:1501-15.

20. Lange T, Vansteelandt S, Bekaert M. A simple unified approach for estimating natural direct and indirect effects. Am J Epidemiol 2012;176:190-5.

21. Dalbeth N, Schauer C, Macdonald P, et al. Methods of tophus assessment in clinical trials of chronic gout: a systematic literature review and pictorial reference guide. Ann Rheum Dis 2011;70:597604.

22. Yamashita S, Matsuzawa Y, Tokunaga K, Fujioka S, Tarui S. Studies on the impaired metabolism of uric acid in obese subjects: marked reduction of renal urate excretion and its improvement by a lowcalorie diet. Int J Obes 1986;10:255-64. 
23. Dessein PH, Shipton EA, Stanwix AE, Joffe BI, Ramokgadi J. Beneficial effects of weight loss associated with moderate calorie/carbohydrate restriction, and increased proportional intake of protein and unsaturated fat on serum urate and lipoprotein levels in gout: a pilot study. Ann Rheum Dis 2000;59:539-43.

24. Neogi T, Hunter DJ, Chaisson CE, Allensworth-Davies D, Zhang Y. Frequency and predictors of inappropriate management of recurrent gout attacks in a longitudinal study. J Rheumatol 2006;33:1049.
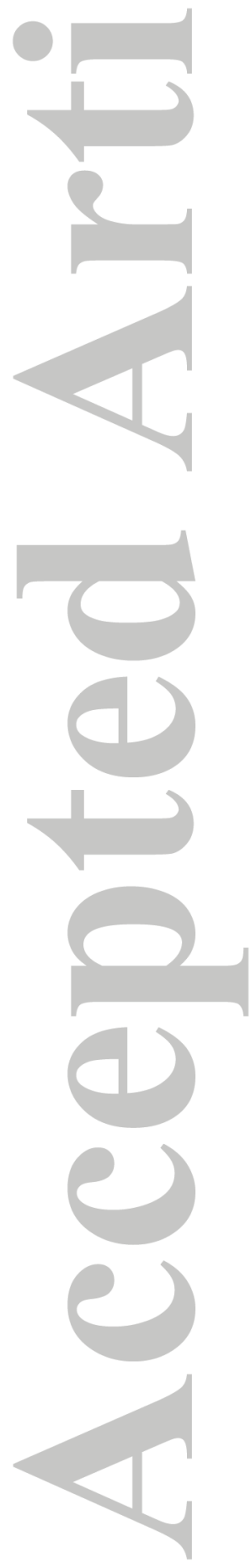

John Wiley \& Sons, Inc.

This article is protected by copyright. All rights reserved. 
Table 1. Baseline Characteristics of Participants without Gout at Baseline in MRFIT According to BMI Category

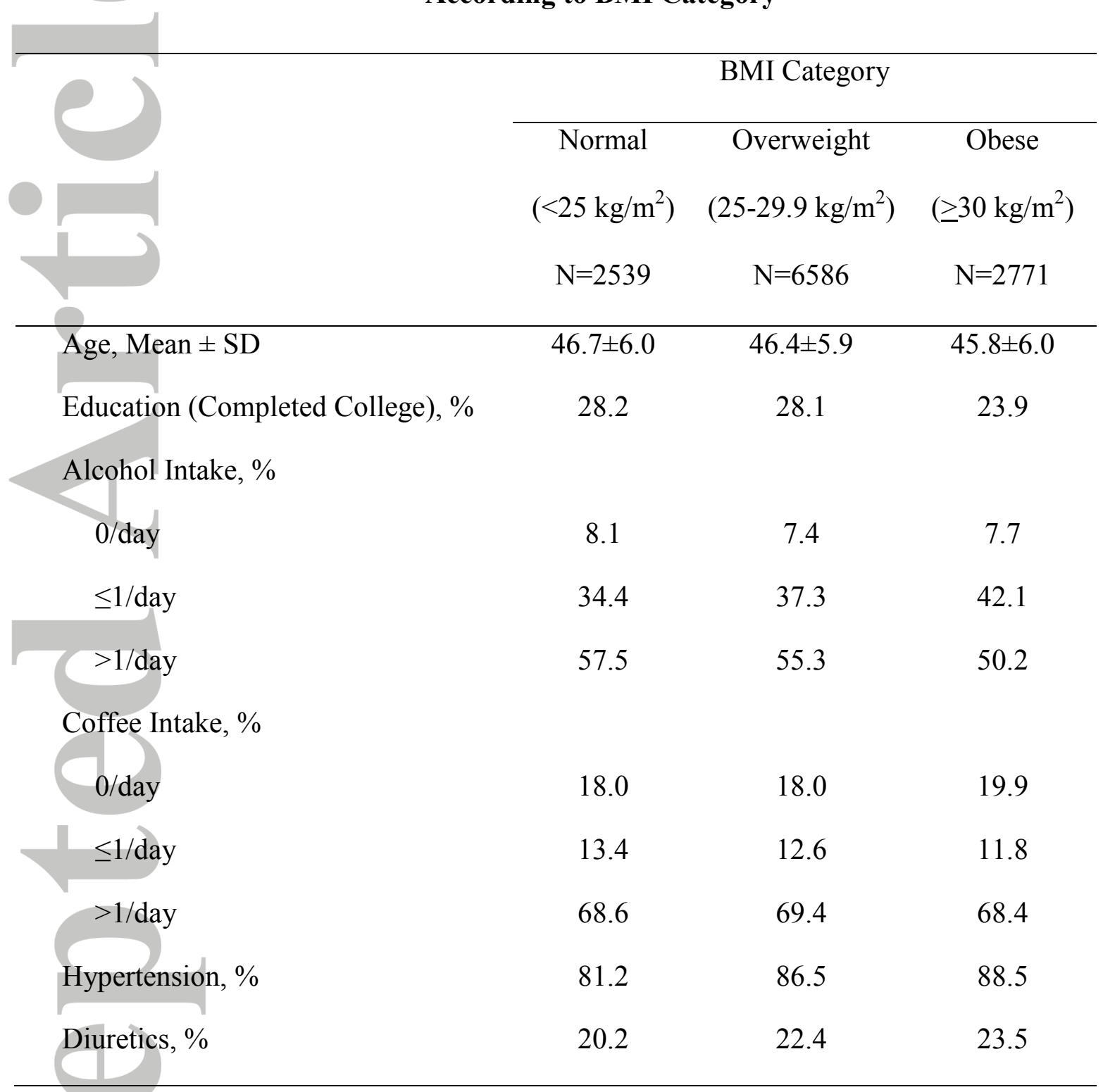


Table 2. Total, Direct, and Indirect Effects of Body Mass Index on Recurrent Gout Attacks among All Participants without Gout at Baseline

\begin{tabular}{|c|c|c|c|c|c|c|}
\hline BMI & Follow-up & N of People with & Recurrent & Total Effect & Indirect Effect & Direct Effect \\
\hline Categories & time (PY) & Recurrent & Gout Attacks & $\mathrm{OR}^{*}(95 \% \mathrm{CI})$ & OR* $(95 \% \mathrm{CI})$ & $\mathrm{OR}^{*}(95 \% \mathrm{CI})$ \\
\hline & & Gout Attacks & Rate (per 1000 PY) & & & \\
\hline Normal & 15,209 & 14 & 0.92 & 1.0 & 1.0 & 1.0 \\
\hline Overweight & 39,344 & 73 & 1.86 & $1.98(1.12,3.51)$ & $1.57(1.17,2.21)$ & $1.27(0.98,1.64)$ \\
\hline Obese & 16,530 & 44 & 2.66 & $2.75(1.50,5.04)$ & $2.83(2.17,3.67)$ & $0.98(0.71,1.34)$ \\
\hline
\end{tabular}

* Adjusted for baseline age, education, alcohol and coffee intake, presence of hypertension, and diuretic use $\mathrm{PY}=$ Person-years

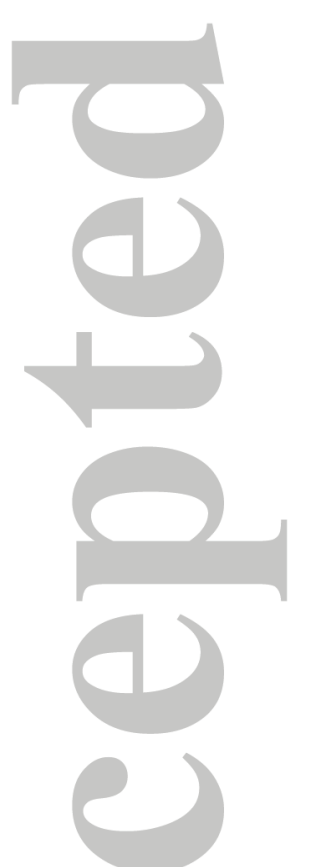


Table 3. Association of BMI Change and the Risk of Recurrent Gout Attacks among Gout Patients

\begin{tabular}{|ccccc}
\hline BMI Change Category* & N of Records & Recurrent & Crude & $\begin{array}{c}\text { Adjusted } \\
\text { OR } \dagger(95 \% \text { CI })\end{array}$ \\
\hline Loss $>5 \%$ & 221 & $14(6.3 \%)$ & $0.71(0.39,1.30)$ & $0.61(0.32,1.16)$ \\
\hline Loss 3.6 to 5 $\%$ & 89 & $8(9.0 \%)$ & $1.00(0.47,2.15)$ & $0.94(0.43,2.06)$ \\
No change (-3.5 to 3.5\%) & 838 & $75(9.0 \%)$ & 1.00 & 1.00 \\
Gain 3.6\% to 5\% & 111 & $13(11.7 \%)$ & $1.35(0.73,2.53)$ & $1.43(0.75,2.72)$ \\
$\quad$ Gain $>5 \%$ & $18(11.4 \%)$ & $1.40(0.79,2.49)$ & $1.60(0.89,2.89)$ \\
\hline P for trend & & & $<0.01$ & $<0.01$ \\
\hline
\end{tabular}

*Relative BMI change $=($ Post BMI - Pre BMI $) /$ Pre BMI

$\uparrow$ Adjusted for BMI, age, education, alcohol and coffee intake, presence of hypertension, and diuretic use measured during the 12 months before the incident gout attack. 


\section{Appendix}

Observational studies of overweight/obesity on recurrent gout attacks among gout patients may have estimated the direct effect (i.e., the effect of overweight/obesity on recurrent gout attacks not through incident gout) rather than the total effect (i.e., the effect of overweight/obesity on recurrent gout attacks through all possible causal mechanisms). The difference between the direct and total effects can be illustrated using a causal diagram (Figure 1a). When investigators examine the association between baseline BMI on the risk of recurrent gout among patients with gout (symbolized by the box around "Incident Gout"), the causal pathway from BMI to recurrent gout attacks via incident gout is blocked, allowing only for the assessment of the effect of BMI on recurrent gout attacks independent of incident gout (i.e., direct effect). Such a direct effect, however, would likely be absent in this study, as a person must first have a gout attack in order to have recurrent gout attacks. It is this incongruence between the research question being asked and the effect being estimated with the usual study design that may explain the obesity paradox seen in prior studies, as the estimated measure of effect may not appropriately address the research question. Furthermore, observational studies of recurrent gout attacks conducted among gout patients could also create a potential selection bias (i.e., collider stratification or index event bias), and such a bias would generally dilute the direct effect, if present, towards the null or in the opposite direction.

Conditioning on an intermediate variable, whether by controlling for incident gout in the analysis or by restricting a study to those with incident gout, would block the indirect effect of baseline obesity on the risk of recurrent gout attacks through obesity's effect on incident gout, and would 
only allow for assessment of the direct effect through mechanisms not involving incident gout.

This direct effect of obesity independent of the first gout attack would be highly unlikely because in order to have recurrent gout attacks, one must first have incident gout. Thus, one would expect a null direct effect of obesity on risk of recurrent gout attacks through other mechanisms. This phenomenon was clearly illustrated with results from our mediation analysis.

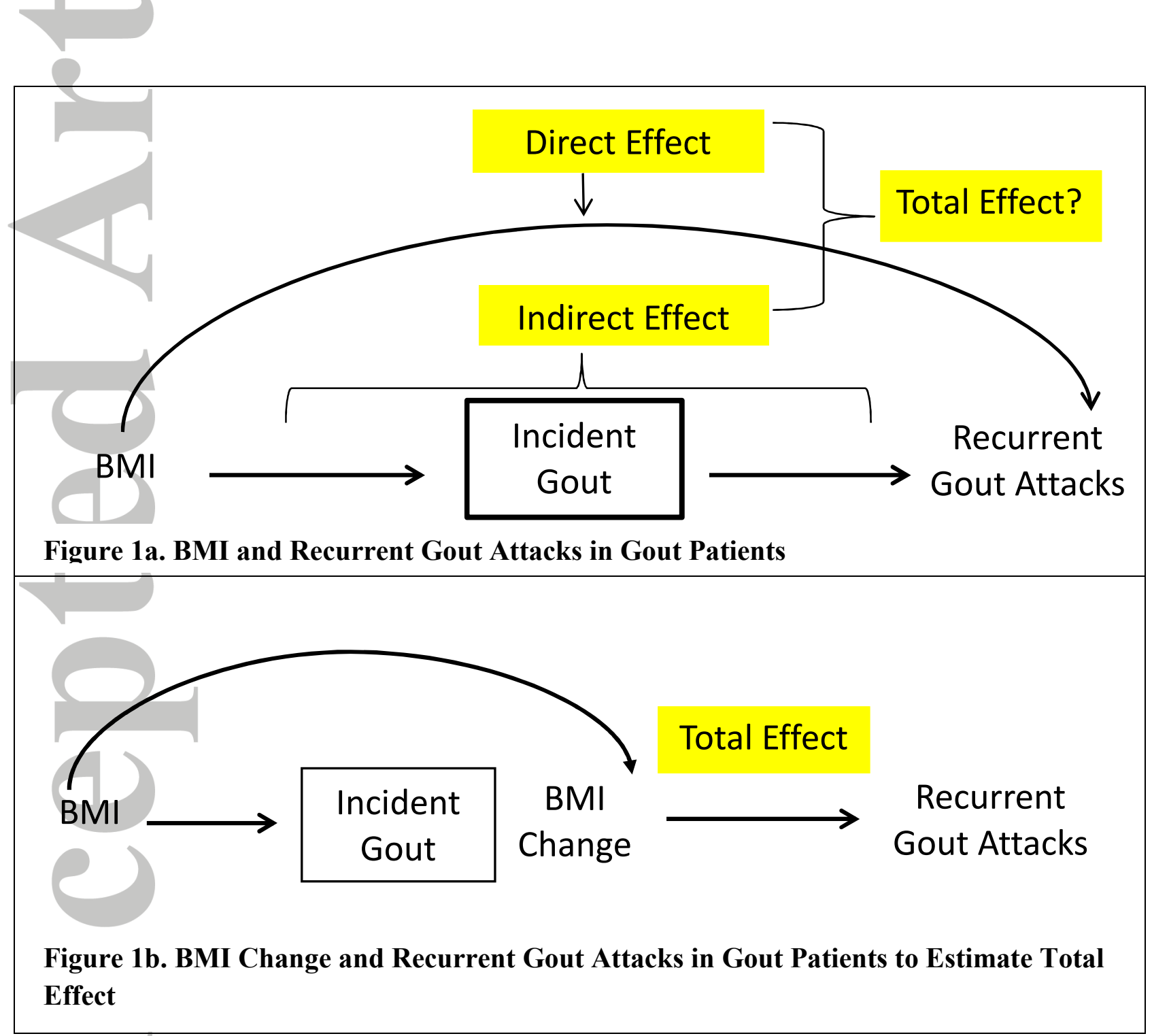


Supplemental Table 1. Association of Baseline BMI and Incident Gout Attack among People Free of Gout at Baseline In the Overall Population

\begin{tabular}{lcccc}
\hline BMI & No. of People & Incident Gout & Crude OR & Adjusted OR* \\
Categories & & $\mathrm{N}(\%)$ & $(95 \% \mathrm{CI})$ & 1.0 \\
\hline Normal & 2539 & $53(2.09 \%)$ & 1.0 & $1.50(1.11,2.02)$ \\
Overweight & 6586 & $208(3.16 \%)$ & $1.53(1.13,2.08)$ & $2.60(1.90,3.57)$ \\
Obese & 2771 & $147(5.30 \%)$ & $2.63(1.91,3.61)$ & \\
\hline
\end{tabular}

* Adjusting for age, education, coffee, alcohol, hypertension, and diuretics at baseline.

Supplemental Table 2. Association of BMI (before incident gout attack) and Recurrent Gout Attack among Gout Patients (Direct Effect)

\begin{tabular}{lcccc}
\hline BMI & No. of Controls & $\begin{array}{c}\text { No. Cases with } \\
\text { Recurrent gout attacks }\end{array}$ & $\begin{array}{c}\text { Crude OR } \\
(95 \% \text { CI })\end{array}$ & $\begin{array}{c}\text { Adjusted OR* } \\
\text { Categories }\end{array}$ \\
\hline Normal & 182 & 17 & 1.00 & 1.00 \\
Overweight & 647 & 71 & $1.20(0.69,2.09)$ & $1.20(0.70,2.14)$ \\
Obese & 482 & 44 & $0.99(0.55,1.76)$ & $0.98(0.53,1.81)$ \\
\hline
\end{tabular}

*Adjusting for age, education, coffee, alcohol, hypertension, and diuretics 12 months before incident gout. 